Revue trimestrielle sur l'image géographique et les formes du territoire

123 | 2018

Varia

\title{
Une géographie à l'école par la pratique artistique
}

\section{Sophie Gaujal}

\section{OpenEdition}

\section{Journals}

Édition électronique

URL : http://journals.openedition.org/mappemonde/477

DOI : 10.4000/mappemonde.477

ISSN : 1769-7298

\section{Éditeur}

UMR ESPACE

\section{Référence électronique}

Sophie Gaujal, « Une géographie à l'école par la pratique artistique », Mappemonde [En ligne], 123 |

2018, mis en ligne le 14 février 2018, consulté le 15 septembre 2020. URL : http://

journals.openedition.org/mappemonde/477

Ce document a été généré automatiquement le 15 septembre 2020.

\section{(c) (i) (2) (2)}

La revue Mappemonde est mise à disposition selon les termes de la Licence Creative Commons Attribution - Pas d'Utilisation Commerciale - Partage dans les Mêmes Conditions 4.0 International. 


\section{Une géographie à l'école par la pratique artistique}

\section{Sophie Gaujal}

\section{Références de la thèse}

GAUJAL S. (2016). Une géographie à l'école par la pratique artistique. Thèse de doctorat de géographie, Paris 7.

1 Le 18 avril 2013 à $9 \mathrm{~h} 50,35$ élèves de classe de Première Économique et Sociale tentaient de rendre leur cour de récréation, délaissée au profit du parvis, à sa fonction première, celle d'une cour accueillant les élèves pendant la récréation. Le temps d'une récréation donc, ils se sont employés à rendre visible un lieu invisible, à faire d'une périphérie un centre, à «inverser les périphéries». Au moyen d'une performance, mobilisant un dispositif sonore et chorégraphique, ils ont ainsi poussé la communauté éducative du lycée à s'interroger sur ses usages de l'espace, voire à les transformer. De cette performance, aboutissement d'une enquête sur leur espace proche menée par la classe en cours de géographie tout au long de l'année, il reste peu de traces. L'invitation qui sera présentée ici est l'une d'entre elles. La thèse qui l'a motivée et dont elle est l'un des résultats, également. 
Figure 1. L'image du mois : un carton d'invitation annonçant une « inversion des polarités » dans un lycée de la banlieue parisienne

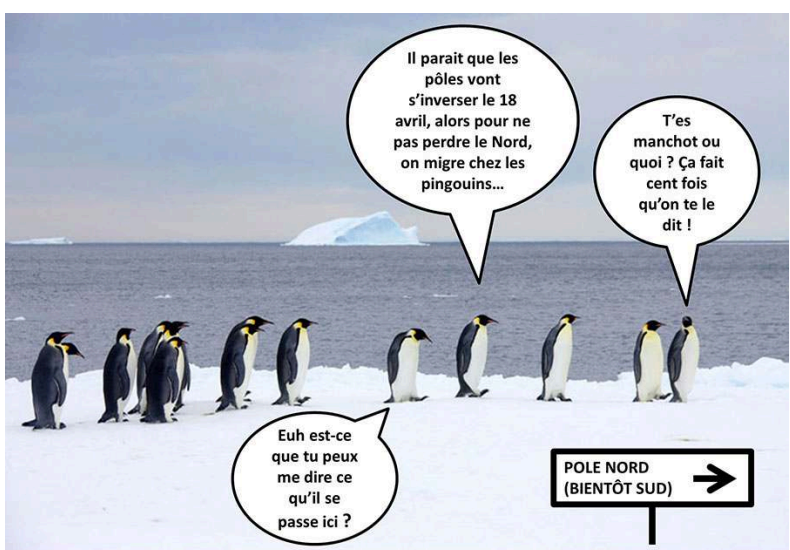

Inversement magnétique des pôles prévu prochainement

L'inversement du champ magnétique terrestre est un phénomène physique et naturel extrêmement rare, ayant lieu une fois tous les 670000 ans environ (le dernier remontant à 780000 ans).

Les scientifiques de la NASA, qui avaient constaté un retard de plus de cent millénaires, annoncent, dans un courrier récent, que la prochaine inversion des pôles est prévu pour le 18 avril 2013 au lycée J.Prévert. Les élèves vous invitent à venir observer cet évènement de $9 \mathrm{~h} 50$ à $10 \mathrm{~h} 15$, dans la cour de récréation le jour même, où davantage d'explications vous seront données.

Bon dérèglement à tous et à toutes !

M@ppemonde, 2018

\section{S. Gaujal, 2016}

2 Cette performance, réalisée par des élèves dans un cadre scolaire, est tout d'abord un exercice de géographie. Plusieurs étapes ont été nécessaires pour le mettre en œuvre. La première a été une enquête menée en cours de géographie sur l'espace du lycée et qui a abouti au constat d'un paradoxe : au moment de la récréation, la cour, pourtant aménagée pour accueillir les élèves, reste vide, alors que le parvis du lycée est saturé, nécessitant plusieurs minutes pour y accéder, alors même que le temps dont disposent les élèves est compté (15 minutes). Il y a donc un écart entre les fonctions du lieu et les usages qui en sont faits. 
Figure 2. 10 h 2, la cour de récréation du lycée

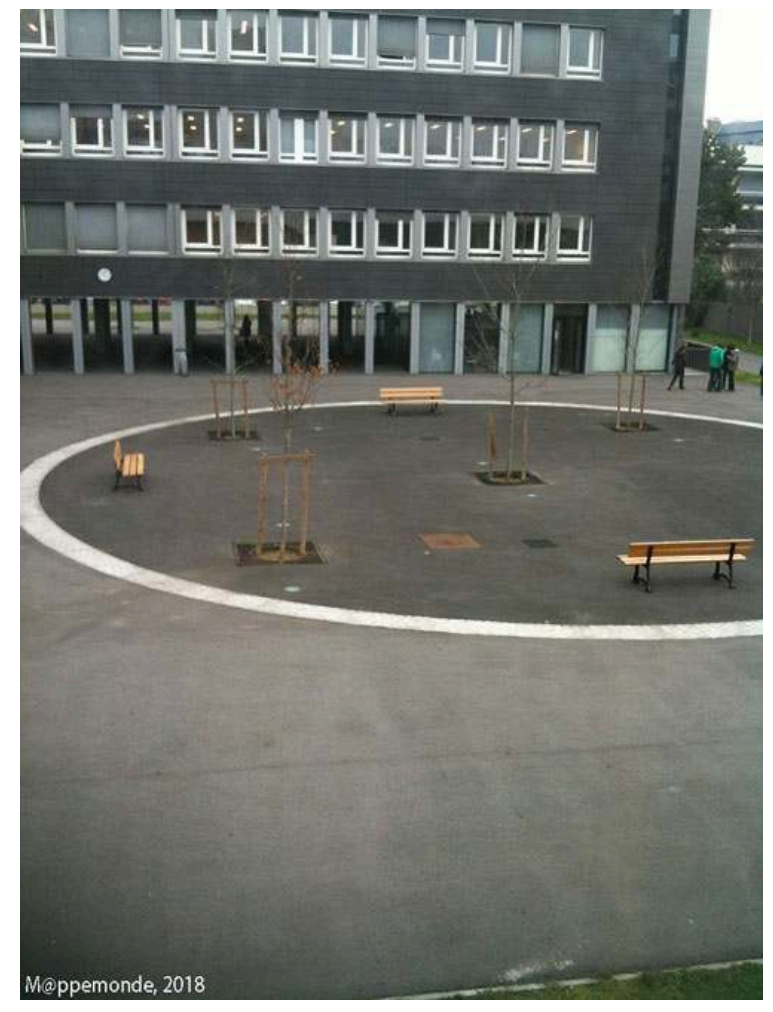

S. Gaujal, 2016

Figure 3. ... au même moment, sur le parvis du lycée

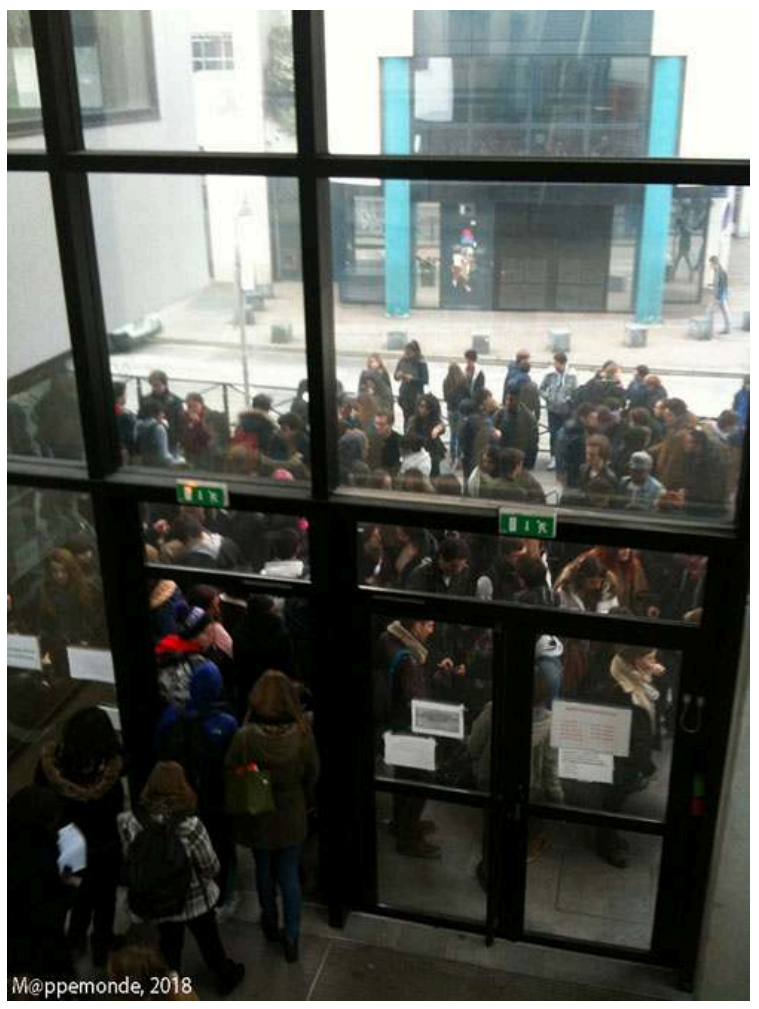

S. Gaujal, 2016 
3 À ce paradoxe plusieurs explications : l'impossibilité de fumer dans la cour, ou encore son organisation en "panoptique inversé » qui fait que, où qu'on soit dans cette cour toute neuve (le lycée a été refait en 2012) et encore insuffisamment arborée, on est au centre des regards. Il ne faut pas moins d'une tempête de neige pour que la cour retrouve, pour quelques instants, sa fonction initiale.

Figure 4. 10 h 2, la cour de récréation, jour de neige

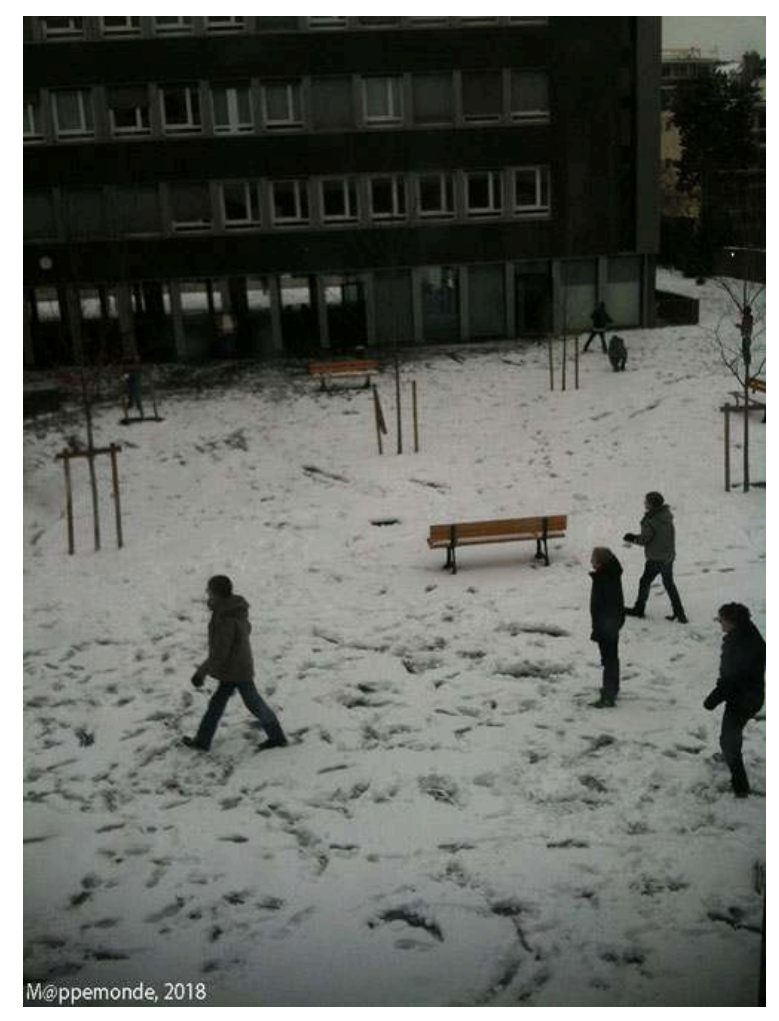

S. Gaujal, 2016

Le constat de ce paradoxe a été le point de départ d'un questionnement géographique, permettant de manipuler avec les élèves différentes notions (marge, périphérie, centre, flux, usages, fonctions, habiter), concepts (différenciation voire discontinuité spatiale), méthodologies (enquêtes quantitatives et qualitatives), et écritures pour formaliser leur analyse (schémas, croquis, photographies). D'espace jusque-là transparent, presque invisible, la cour de récréation a acquis, au terme de cette enquête, un nouveau statut, celui d'un espace marginal, comme le montre le schéma ci-dessous. 
Figure 5. Plan du lycée (d'après le plan-masse) : la cour de récréation, un espace marginalisé

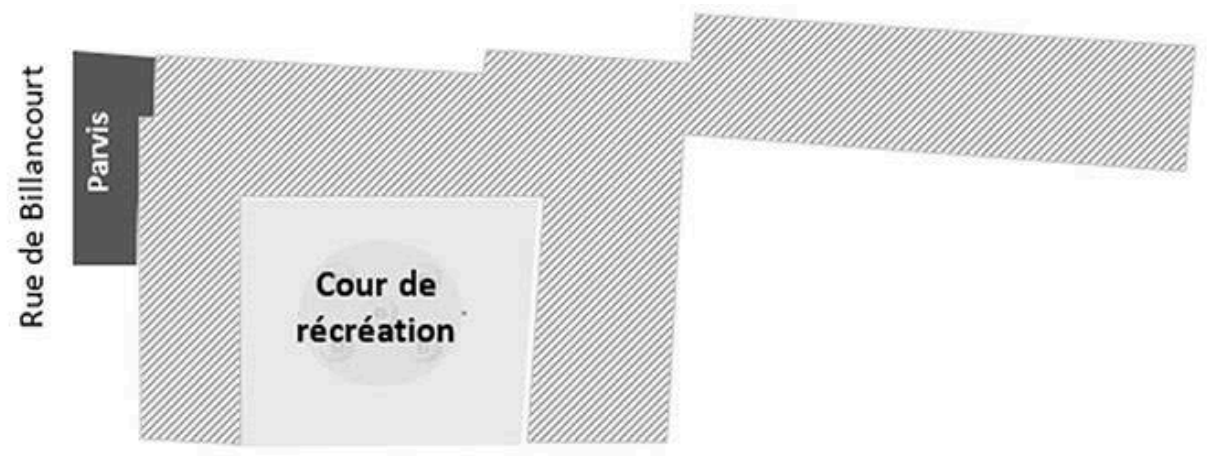

Le lycée, un bâtiment dont le corps principal est organisé en « $U$ » autour de la cour, selon un principe qui peut évoquer celui d'un panoptique inversé.

La cour, un espace aménagé pour accueillir les élèves pendant la récréation, mais marginalisé dans la pratique ...

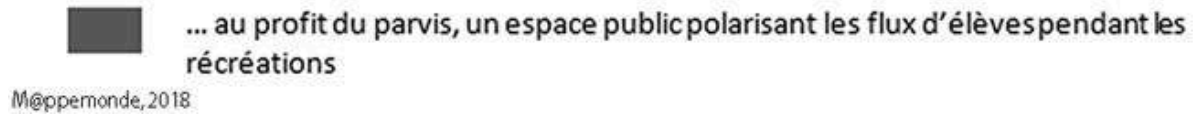

S. Gaujal, 2016

De là a surgi un nouveau questionnement : était-il possible d'inverser les polarités ? De faire de la cour, le temps d'une récréation, un pôle, et du parvis une marge ? Et, au-delà, de permettre à la classe de communiquer ses résultats au reste de la communauté éducative, et de rendre ce lieu visible aux yeux de tous? D'analystes de l'espace, les élèves étaient ainsi invités à devenir créateurs d'espace, à agir sur lui, à tester sa plasticité. Commence alors une deuxième étape de conception d'une action sur l'espace. Pour y parvenir, différents exemples d'interventions d'artistes contemporains, « œuvrant d'art» l'espace (Volvey, 2012), comme celles de Christo et Jeanne Claude, Ernest Pignon Ernest, Till Roeskens, La Luna, ont été présentées aux élèves. J'ai également sollicité l'aide du professeur d'arts du son et musicien performeur, Sébastien Branche. Le carton d'invitation (figure 1) rend compte du projet auquel cela a abouti : il s'agira d'inverser les polarités, le temps d'une "récré'action». Les pingouins sont avertis : bientôt le pôle Nord va devenir le pôle Sud (figure 6).

Figure 6a. Un constat : la cour de récréation marginalisée au profit du parvis Figure 6b. L'objectif : inverser les polarités, le temps d'une récréation
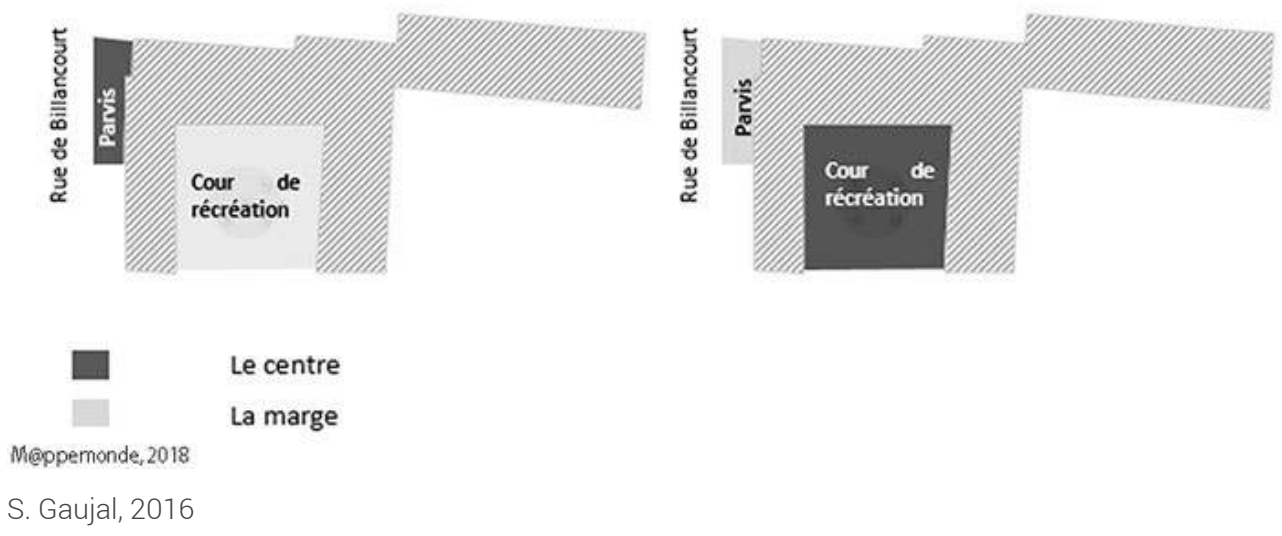

retenti dans la cour des sons que l'on n'y avait jamais entendus jusque-là, préalablement enregistrés sur le parvis par Sébastien Branche, d'adolescents qui discutent, qui chantent, qui jouent, tandis que les élèves de la classe s'y égayaient.

Ce média ne peut être affiché ici. Veuillez vous reporter à l'édition en ligne http:// journals.openedition.org/mappemonde/477

Depuis les salles de classe, disposées nous l'avons montré plus haut en «U » autour de la cour, les autres élèves ont alors découvert le spectacle. Intrigués, alertés par un faux signal de récréation (la «vraie » récréation commençait 5 minutes plus tard), ils ont quitté leur salle de classe pour venir voir le spectacle de plus près, plaçant la cour, le temps d'une récréation, au centre de l'attention et la rendant à sa fonction initiale.

Figure 7. 18 avril 2013, la cour au centre de l'attention, le temps d'une récréation

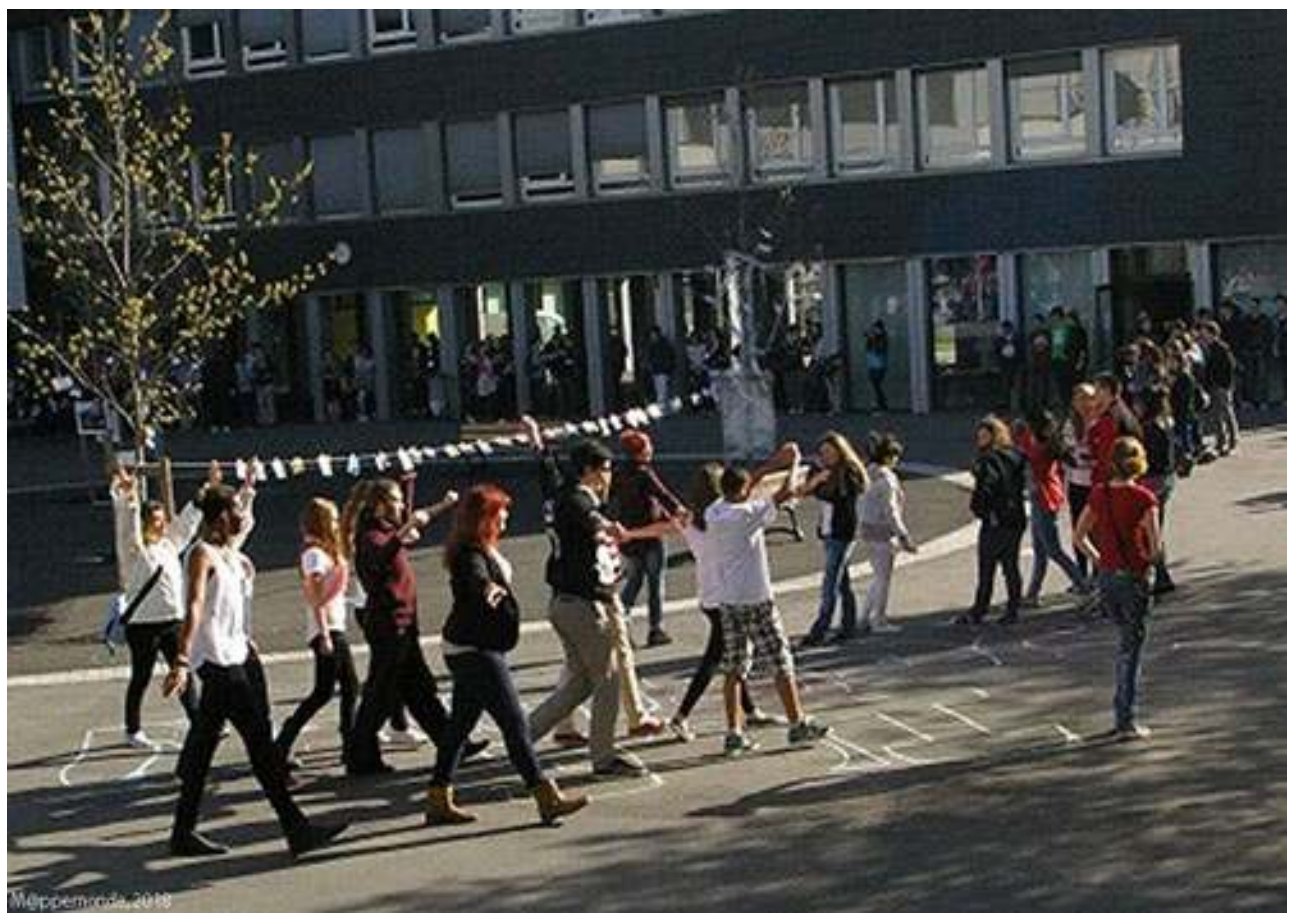

S. Gaujal, 2016

Cet exercice n'aurait cependant pas été conçu par la praticienne que je suis sans le soutien de la recherche. Il est, en effet, autant qu'un exercice proposé aux élèves, une expérimentation réalisée dans le cadre d'une recherche en didactique de la géographie depuis une posture de praticienne-chercheure, c'est-à-dire d'enseignante engagée dans une recherche, prenant sa pratique à la fois comme objet et comme terrain. Elle s'appuie sur un cadre théorique pluriel, composé de recherches menées en didactique de l'histoire-géographie et en géographie de l'art (Gaujal, 2016). Les premières m'ont permis de caractériser le fonctionnement de la classe ordinaire en cours de géographie, privilégiant le cours magistralo-dialogué, dans lequel les interactions sont limitées (Audigier, 1993 ; Tutiaux-Guillon, Mousseau, 1998; Fontanabona, Thémines, 2005 ; Lautier, Allieu-Mary, 2008), et portant majoritairement sur la petite échelle, celle des États et des aires régionales, négligeant l'échelle micro-spatiale des pratiques du 
quotidien (Clerc, 2012). Loin de ces pratiques ordinaires, j'ai donc entrepris, via une recherche-développement, de concevoir, expérimenter et diffuser des exercices permettant aux élèves d'articuler leurs pratiques de l'espace (la géographie spontanée) avec les concepts de la géographie (la géographie raisonnée). C'est pour les concevoir que j'ai eu recours aux travaux menés en géographie de l'art. Ils ont permis de caractériser les manières de faire des artistes contemporains, en proie à un tournant spatial, et, de manière symétrique, sur les manières d'interroger l'espace par les géographes, en proie à un tournant esthétique (Volvey, 2012), les conduisant, par exemple, à se tourner vers la cartographie sensible (Olmedo, 2015). Les exercices que j'ai conçus (visite sensible à la suite de Médéric Briand [2014], performance, photographie, cartographie sensible) ont eu pour objectif de transposer ces manières de faire dans un cadre scolaire, avec l'hypothèse qu'elles peuvent favoriser l'apprentissage de savoirs géographiques par les élèves. La créativité que cela a engendrée permet de caractériser cette recherche comme une recherche-création.

Un mot, pour conclure, de la suite donnée à ces recherches. Soutenue en 2016, la thèse n'en est qu'un jalon. Membre d'un groupe de recherche en didactique de la géographie sur le raisonnement en géographie, je poursuis mes recherches en direction d'une géographie expérientielle. En 2016-2017, à nouveau, la cour de récréation a servi de terrain d'investigation, pour étudier cette fois les dynamiques de cet espace. Jusque-là marge, le plan Vigipirate, qui interdit les rassemblements devant les établissements scolaires, en a fait un nouveau centre. L'autorisation pendant un temps d'y fumer et l'installation d'un algeco au centre de la cour pour accueillir de nouvelles classes ont alors eu raison des réticences des élèves. Une carte sensible, produite ci-dessous, est venue matérialiser les conclusions de cette enquête.

Figure 8a. Carte sensible du lycée, 2016-2017, classe de Première ES

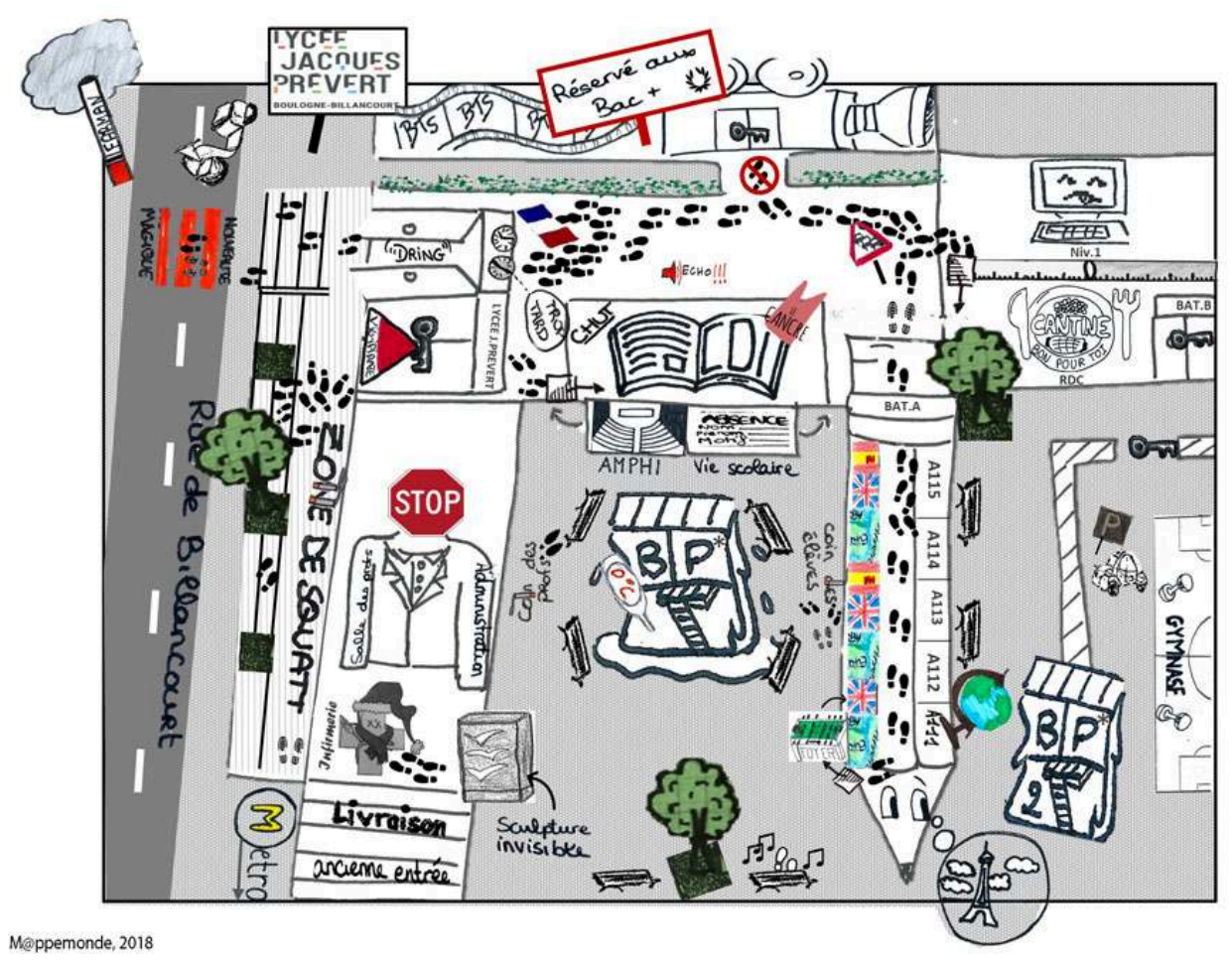

S. Gaujal, 2016 
Figure 8b. Carte sensible du lycée, 2016-2017, classe de Première ES

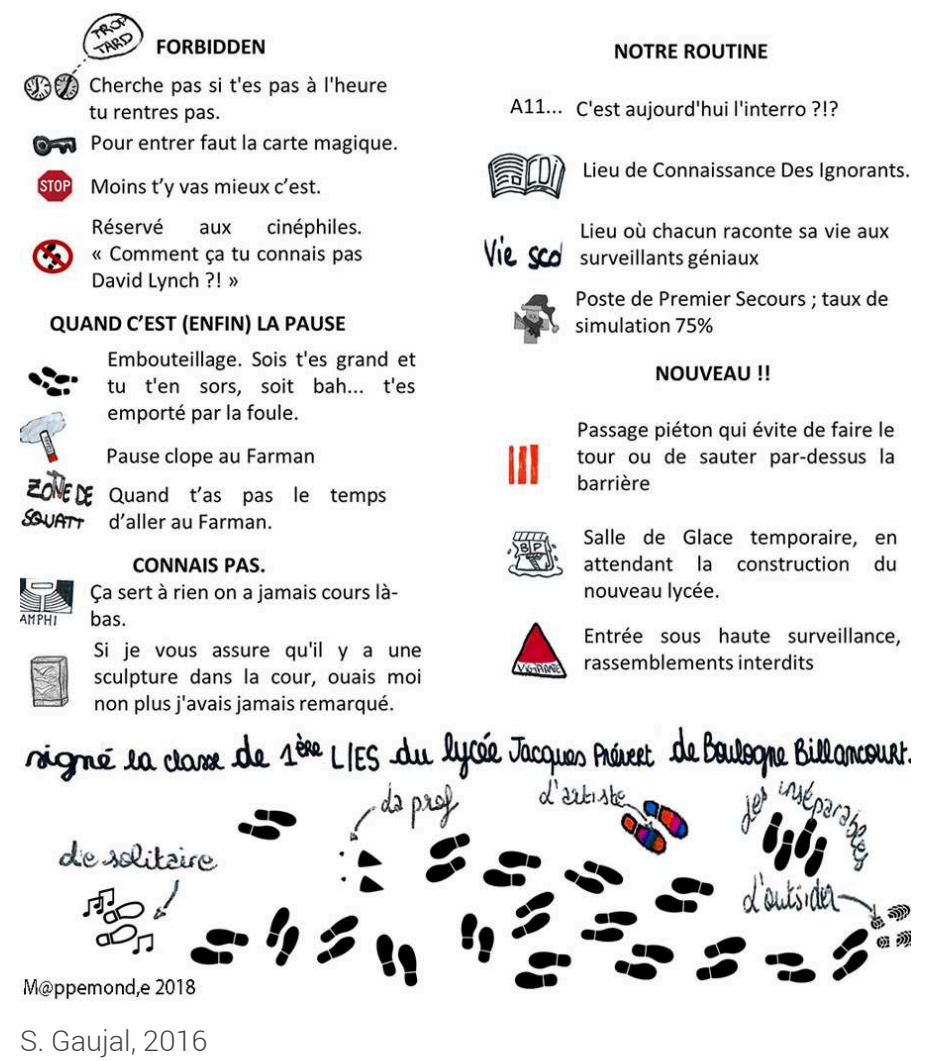

\section{BIBLIOGRAPHIE}

AUDIGIER F. (1993). Les représentations que les élèves ont de l'histoire et de la géographie. À la recherche des modèles disciplinaires, entre leur définition par l'institution et leur appropriation par les élèves. Thèse de doctorat en sciences de l'éducation, Paris 7, dir. H. Moniot.

BRIAND M. (2014). La géographie scolaire au prisme des sorties : pour une approche sensible à l'école élémentaire. Thèse de doctorat en géographie, Université de Caen Basse-Normandie. En ligne CLERC P. (dir.) (2012). Géographies. Épistémologie et histoire des savoirs sur l'espace. Paris : Armand Colin, 312 p. ISBN 9782301001405

FONTANABONA J., THEMINES J.-F. (2005). Innovation et histoire-géographie dans l'enseignement secondaire : analyses didactiques. Lyon : INRP.

GAUJAL S. (2016). « Peut-on faire des élèves, via une démarche artistique, des acteurs-créateurs de l'espace qu'ils habitent ? » In THÉMINES J.-F. et DOUSSOT S., Acteurs et actions, perspectives en didactiques de l'histoire et de la géographie. Caen : PUC. ISBN 978-2-84133- 835-1 
LAUTIER N., ALLIEU-MARY N. (2008). « La didactique de l'histoire ». Revue française de pédagogie. Recherches en éducation, $\mathrm{n}^{\circ} 162$ (janvier), p. 95-131.

OLMEDO E. (2015). Cartographie sensible. Tracer une géographie du vécu par la recherche-création. Thèse de doctorat de géographie, Paris 1, dir. J.-M. Besse.

TUTIAUX-GUILLON N., MOUSSEAu M.-J. (1998). « Dans la classe, l'influence du modèle pédagogique sur l'explicatif ». In AUDIGIER F.(dir.), Contributions à l'étude de la causalité et des productions des élèves en histoire et en géographie, Paris : INRP, p. 33-67.

VOLVEY A. (2012). Transitionnelles géographies: Sur le terrain de la créativité artistique et scientifique. HDR, Lyon : Université Lumière-Lyon 2.

INDEX

Mots-clés : prix de thèse 2017 